\title{
Coulomb Correlations and Pseudo-gap Effects in a Pre-formed Pair Model for the Cuprates
}

\author{
Jiri Maly, K. Levin and D. Z. Liu \\ James Franck Institute, University of Chicago, Chicago, IL 60637
}

(Submitted to Physical Review B August 27, 1996)

\begin{abstract}
We extend previous work on pre-formed pair models of superconductivity to incorporate Coulomb correlation effects. For neutral systems, these models have provided a useful scheme which interpolates between BCS and Bose Einstein condensation with increasing coupling and thereby describes some aspects of pseudo-gap phenomena. However, charge fluctuations (via the plasmon, $\omega_{p}$ ) significantly modify the collective modes and therefore the interpolation behavior. We discuss the resulting behavior of the pseudo-gap and thermodynamic quantities such as $T_{c}, \chi$ and $C_{v}$ as a function of $\omega_{p}$.
\end{abstract}

The role of the pseudo-gapl in the high $T_{c}$ cuprates is emerging as an important indicator of the nature of the superconductivity as well as the normal state. There are two widely discussed but competing explanations for pseudo-gap effects but no clear and decisive experiments to support one scenario over the other. Early observa tions associated the pseudo-gap with magnetic pairinge above $T_{c}$ (often called the "spin gap"). It is now clear, however, that some form of normal state pairing is seen in photoemission as well as charge transport data. Moreover, at least in the photoemission data the pseudo-gap appears to have the $d$-wave symmetry 1 of the ordered state and this leads naturally to the asspciation of this "gap" with precursor superconductivity. 3 - This second scenario is further supported by the observation of low dimensionality and short coherence lengths in high $T_{c}$ superconductors, which suggests important deviations from ideal mean field or BCS transitions. Indeed, the approach of the present paper assumes the precursor superconductivity scenario, in large part because it is important to establish, at least as a base-line, the extent to which such superconducting "fluctuation" effects may be responsible for pseudo-gap behavior.

Among those models which subscribe to a precursor superconductivity scenario there are additionally two rather distinct viewpoints. Emery and Kivelsonl have argued that the pseudo-gap state of the cuprates is similar to that observed in granular films where phase coherence is not fully established, although large regions of the material have a well established superconducting amplitude. Because it is small, in some sense, in the cuprates their approach focuses on $n / m^{*}$ or alternatively on the plasma frequency $\omega_{p}$ as the fie "phase stiffness" parameter. Alternatively, others 3 it 6 have focused on the observed small size of the superconducting correlation length $\xi$ to argue for important corrections to BCS the ory associated with pre-formed or nearly-formed pairs 8 which exist well above $T_{c}$ and therefore give rise to significant pseudo-gap effects. The present paper is based on the viewpoint that in the cuprates the characteristic parameter of the charge degrees of freedom, $n / m^{*}$ or equivalently $\omega_{p}$, should be treated on a relatively equal footing with the correlation length, $\xi$.
To study the role of Coulomb interactions on pseudogap phenomena, we adopt a natural microscopic framework which incoporates charge fluctuations into theories which treat the cross-over from BCS pairing to BoseEinstein condensation (BEC) of pre-formed pairs. In neutral systems, this fross-over has been studied by a variety of investigators. 3 . Numerical simulation studies, which have been performed in the context of attractive Hubbard models, include, in principle, all diagrammatic contributions. On the other hand, analytical work has mostly been confined to the T-matrix approximation. The issue of non-conserving and conserving T-matrix schemes has been widely discussed in the literature 6 in the context of the BCS-BEC cross-over problem. In the original work of Nozières and Schmitt-Ripk a nonconserving approach was used. Recent work 6 on neutral systems has extended this scheme using a T-matrix approximation which satisfies global conservation laws and in the process introduces renormalized Green's functions into the generalized susceptibilities. In the charged system, as a consequence of gauge invariance, the analogue renormalized susceptibilities must then appear in the particle-hole channel. As has been known for some time 9 however, the collective mode spectrum is then treated incorrectly at this level of approximation and a more sophisticated scheme is needed. In order to avoid this complexity and to develop an intuitive understanding of the effects of charge, however, we restrict the analysis, in this paper, to the more familiar scheme introduced by Nozières and Schmitt-Rink and defer consideration of a fully conserving formalism. We note, however, that our formulation will be locally conserving and in the case of charged systems this approximation does not yield qualitatively different physics from that expected using a globally conserving approach. Furthermore, it is our contention that mode-mode coupling effects, which are ignored in all T-matrix based schemes, will ultimately lead to important insights which we will discuss in a future paper.

The Hamiltonian under consideration contains an attractive interaction $V_{\mathbf{k}, \mathbf{k}^{\prime}}$, parameterized by a coupling constant $g$, as well as long range Coulomb terms. For definiteness we take the same separable pairing poten- 
tial, $V_{\mathbf{k}, \mathbf{k}^{\prime}}=g v_{\mathbf{k}} v_{\mathbf{k}^{\prime}}$ where $v_{\mathbf{k}}=\left(1+k^{2} / k_{0}^{2}\right)^{-1 / 2}$, as was used initially by Nozières and Schmitt-Rink. Within this model, the pairing energy scale (or "Debye frequency") is the Fermi energy. We assume a three dimensional free electron model for the electrons and defer discussion of anisotropy effects until later in the text. It is assumed that in the cuprates there is sufficient inter-layer hopping so that a strictly two dimensional model and its associated Kosterlitz-Thouless transition is not the appropriate starting point. We generalize the path integral formulation of Ref. 1 , replacing the usual fermionic fields by Nambu spinors, $\psi_{\mathbf{k}}^{\dagger}=\left(c_{\mathbf{k} \uparrow}^{\dagger}, c_{-\mathbf{k} \downarrow}\right)$, and decoupling in four real fields given by $\eta_{i}=\psi^{\dagger} \tau_{i} \psi\left(\tau_{i}, i=0 \ldots 3\right.$, are the identity and three Pauli matrices). The interaction in the off-diagonal channels $(i=1,2)$ is the pairing interaction while the Coulomb term appears in the diagonal channels $(i=0,3)$. Finally, the field $\eta_{0}$ may be eliminated by a suitable gauge transformation and the thermodynamic potential $\Omega$ is computed for the remaining degrees of freedom at the Gaussian approximation level,

$$
\begin{aligned}
\Omega & =\Omega_{0}+\frac{T}{2} \sum_{\mathbf{q}, \nu_{m}} \ln \operatorname{det}\left(\Gamma_{\mathbf{q}, \nu_{m}}^{-1}\right)-\frac{n_{f}}{2} \sum_{\mathbf{q}} V_{\mathbf{q}}^{(3)}, \\
\Gamma_{\mathbf{q}, \nu}^{(i, j)-1} & =\delta_{i, j}-T V_{\mathbf{q}}^{(i)} \sum_{\mathbf{k}, \omega_{l}} \operatorname{tr}\left(\tau_{i} \mathcal{G}_{\mathbf{k}+\mathbf{q} / 2, \omega_{l}} \tau_{j} \mathcal{G}_{\mathbf{k}-\mathbf{q} / 2, \omega_{l}-\nu_{m}}\right) v_{\mathbf{k}}^{(i)} v_{\mathbf{k}}^{(j)} .
\end{aligned}
$$

Here $\Omega_{0}$ is the usual contribution from non-interacting fermions and $n_{f}$ denotes the corresponding number density of free fermions. In the interaction $V_{\mathbf{q}}^{(i)}=g, v_{\mathbf{k}}^{(i)}=$ $v_{\mathbf{k}}$ for $i=1,2$, and $V_{\mathbf{q}}^{(3)}=4 \pi e^{2} / \kappa q^{2}, v_{\mathbf{k}}^{(3)}=1$. Finally, $\mathcal{G}_{\mathbf{q}, \omega}$ is the single particle Green's function in Nambu space and $T$ is the temperature, while $\nu_{m}$ and $\omega_{l}$ are the even and odd Matsubara frequencies, respectively. Above $T_{c}$ the fluctuation propagator $\Gamma$ is diagonal; thus all three channels (the particle-particle, particle-hole and uncorrelated fermions) contribute additively to $\Omega$ as well as to the various derived thermodynamic quantities which we calculate below.

In the present formalism, $\theta$ the transition temperature is obtained from the BCS gap equation with a self consistently determined chemical potential, $\mu$, obtained from the condition $n_{t o t}=-\partial \Omega / \partial \mu$. The resulting coupled equations are solved numerically for $\mu$ and $T_{c}$. In the limit of arbitrarily large $g$ the effects of the Coulomb interaction drop out, since mode-mode coupling is neglected at the Gaussian level, so that $T_{c}$ is given by the ideal BEC temperature. At small $g$, the collective mode contribution to $\mu$ becomes arbitrarily small and the BCS limit is approached, alleit with a Coulomb renormalized chemical potential. 10 When the superconducting coupling constant $g$ vanishes, the above form for $\Omega$ reflects the plasmon contribution and reduces to that of the usual Coulomb gas. 10 The Gaussian approximation to $\Omega$ gives an RPA-like treatment of the collective modes and so provides a reasonable scheme for interpolating between these two limits. It should be noted, however, that Coulomb pseudo-potential effects (which would act to renormalize $g$ ) are, for simplicity, not included in our calculations. Here we focus principally on the effects introduced by the long range Coulomb interaction (which enters via the parameter $\left.\omega_{p}^{2}=4 \pi n e^{2} /\left(m^{*} \kappa\right)\right)$.
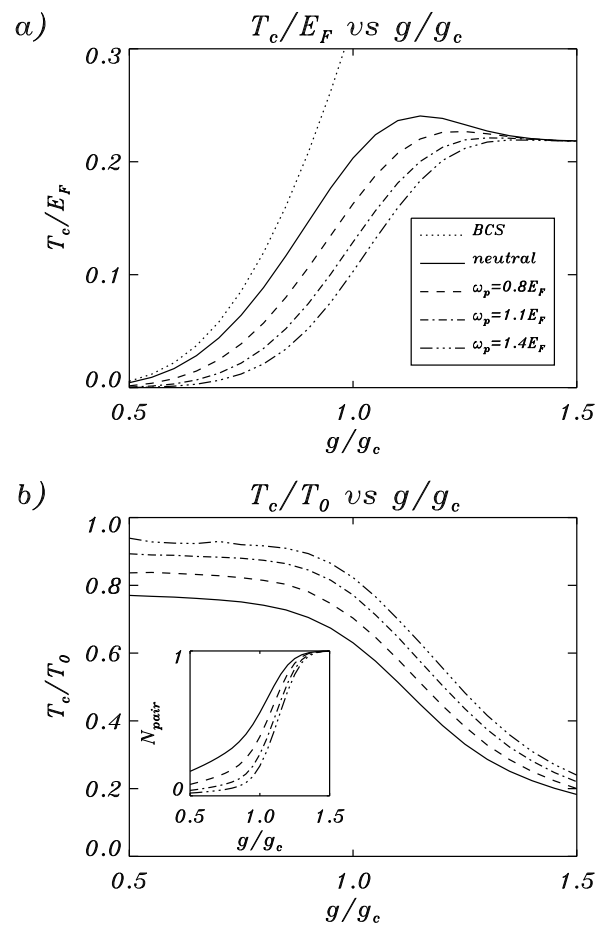

FIG. 1. (a) The variation of $T_{c}$ as a function of coupling constant in the BCS and Gaussian approximation theories for neutral and charged systems with increasing plasma frequencies. (b) $T_{c}$ normalized by the Coulomb mean field result (see text) $T_{0}$ for the same plasma frequencies as in (a). The inset in (b) plots the number of paired electrons vs $g / g_{c}$ for the same parameter set.

To illustrate the effects of charge fluctuations we plot, in Fig. 11 a, $T_{c}$ as a function of $g / g_{c}$ for various values of the plasma frequency. The dotted line represents the BCS result (for the neutral system) and the solid line is the corresponding (neutral) transition temperature which includes Gaussian fluctuations. The remaining curves from left to right demonstrate the effects of increasingly large $\omega_{p}$. For the purposes of focussing on charging effects only we fix $E_{F}$ and $k_{F} / k_{0}$; in this way all the Gaussian derived curves have the same high $g$ asymptote. Moreover, with these assumptions the neutral system reference curve is unchanged as $\omega_{p}$ is varied. Two effects of Coulomb interactions can be observed in Fig. 11a: (i) $T_{c}$ decreases with increasing $\omega_{p}$, and (ii) the non-monotonic behavior as a function of $g$ found for the neutral case (which is believed to be unphysical) 6 progressively disappears with increasing $\omega_{p}$.

The first observation, which is perhaps the more important, is a consequence of the fact that an attraction due to Coulomb interactions in the particle-hole channel reduces the effectiveness of the attraction in the pairing channel. We illustrate this first point more directly in the 
inset of Fig. [1 b, which plots the number of superconducting pairs as a function of increasing $\omega_{p}$. This effect may seem counter to the expectation that systems with larger $\omega_{p}$ will have reduced $T_{c}$ suppression (i.e. larger $T_{c}$ ) due to phase fluctuations. However, when the appropriate reference temperature is used, Coulomb interactions are found to lead to better agreement with mean field theory; in this sense superconducting fluctuations are, indeed, suppressed by Coulomb interactions. We plot in the main portion of Fig. 1 $1 \mathrm{~b}$ the ratio of $T_{c}$ to the critical temperature $T_{0}$ obtained by neglecting pair fluctuations (but including Coulomb effects) for the same range of plasma frequencies as in Fig. 17a. As can be seen, the larger the plasma frequency, the higher the ratio $T_{c} / T_{0}$ and thus the better the agreement with a mean field treatment of the pairing channel.

a)

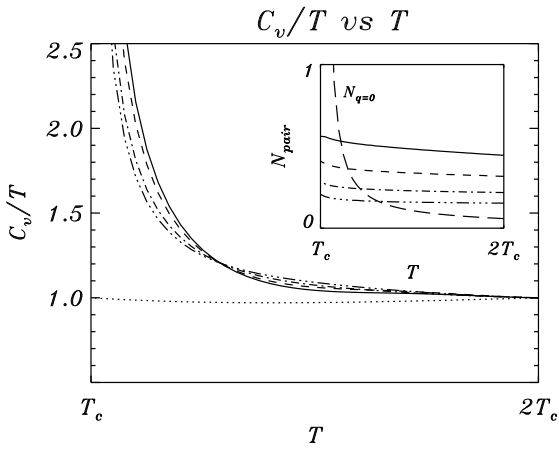

b)

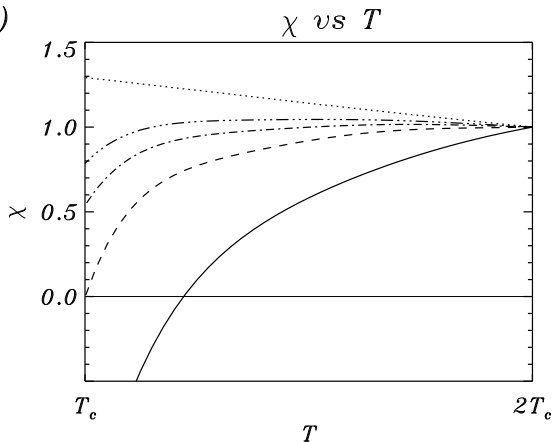

FIG. 2. Temperature dependent specific heat (a) and spin susceptibility (b) for the same parameters as in Figures 1. The dotted lines are the BCS results (with slightly temperature dependent chemical potential). The inset in (a) plots the total number of pairs vs $T$, along with the number at $q=0$.

A more convenient way of illustrating fluctuation effects and the associated role of the plasma frequency, however, is to study thermodynamic properties directly. Here pseudo-gap effects enter as precursor superconducting contributions in, for example, the specific heat and spin susceptibility. As in Fig. 1 b these fluctuation effects are expected to weaken as the plasma frequency increases and mean field behavior is restored. We plot $C_{v}$ and $\chi$ in Fig. 2 for the same parameter set as in Fig. 1, with $g / g_{c}$ set equal to unity. This choice of coupling strength is consistent with the observation of relatively short coherence lengths in the cuprates and with the claim that high $T_{c}$ compounds lie close to the bound state limit. Here the dotted lines again represent the BCS value (with a properly $T$ dependent $\mu$ ). As is appropriate 11 for Gaussian fluctuation theories, the specific heat varies as $\left(T-T_{c}\right)^{-1 / 2}$. Comparison with the neutral (solid) reference curve shows that the effects of variable $\omega_{p}$ are not as evident in the specific heat as in the spin susceptibility. Similarly, deviations from mean-field behaviour appear at higher temperatures in $\chi$ than in $C_{v}$.

It is worth noting that in the present formulation there is no well defined "onset temperature" for the appearence of a pseudo-gap, as might exist if a sharp phase transition occured at some temperature $T^{*}$ above $T_{c}$. Moreover in discussing the onset of fluctuations, it should be stressed that their appearance is unrelated to the temperature dependence of the total number of fluctuations or preformed pairs. As shown in the inset of Fig. 2a, for fixed $\omega_{p}$, the total number of pairs is relatively constant up to temperatures many times higher than $T_{c}$; however, their distribution shifts to lower momenta as $T_{c}$ is approached and long range coherence is established. Detailed calculations indicate that varying $\omega_{p}$ does not alter the critical behaviour in the pairing channel as $T_{c}$ is approached; the narrowing of the pseudo-gap region with increasing $\omega_{p}$ is a result of the smaller relative contribution that pairing fluctuations make to the thermodynamics as Coulomb correlations become more dominant. Finally, it should also be noted that the neutral system yields unphysical thermodynamic behavior at smaller coupling constants than when Coulomb effects are included. This is illustrated by the negative values of $\chi$ indicated in the figure. Such unphysical behaviour has been shown to result from the non-conserving nature of the approximations used.12

While the above figures were designed to illustrate the effect of Coulomb correlations, they do not fully represent the physical system in which variations in the plasma frequency are necessarily associated with changes in the electronic energy scale. In reality both $\omega_{p}$ and $E_{F}=k_{F}^{2} / 2 m^{*}$ depend on similar combinations of the carrier density $n$ and effective mass $m^{*}$. As the insulator is approached $\omega_{p}$ decreases as $\omega_{p}^{2} \approx x$ (where $x$ denotes the number of doped holes); however, whether one assumes a Fermi liquid $(n \approx 1+x)$ or non-Fermi liquid $(n \approx x)$ approach to the insulator, it follows necessarily that the electronic energy scale $E_{F}$ must also vanish as the hole concentration $x$ approaches 0 . In scenarios based on electronic pairing mechanisms, therefore, it is difficult to escape the conclusion that the onset temperature for coherent superconducting fluctuations, $T^{*}$, should also become small as the insulator is approached. Our numerical calculations of $C_{v}$ and $\chi$ exhibit this effect, principally because ours is a single energy scale theory: both $T^{*}$ and $T_{c}$ derive from the same pairing mechanism. This behavior is in contrast to experimenti where even in highly underdoped systems $T^{*}$ is of the order of $100 \mathrm{~K}$ or more. Large $T^{*}$ seems to be most naturally associated with a high energy scale in the insulating parent com- 
pound, such as a magnetic energy. 2 However, in such a scenario it then becomes problematic to understand how the other important energy scale $8 \omega_{p}$ enters to determine $T_{c}$. We speculate that a cross-over from three to two dimensionality may play some role in lowering $T_{c}$ at low doping concentrations where enhanced (quasi-2d) critical fluctuation effects are most apparent.13

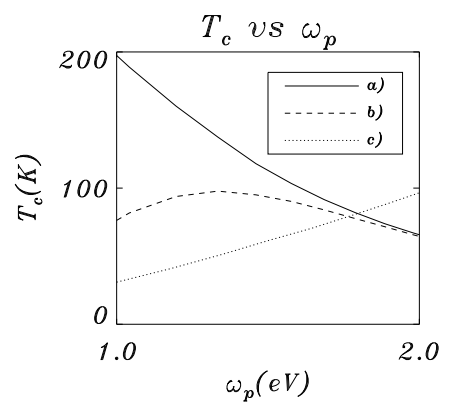

FIG. 3. Variation of $T_{c}$ with $\omega_{p}$ (in units of $e V$, where optimal doping corresponds to $\omega_{p} \approx 1.2 \mathrm{eV}$ ) with $E_{F}$ held fixed (curves (a) and (b)) as well as using self-consistently determined $E_{F}$ (curve(c)). Curve (b) corresponds to $2+\epsilon$ dimensions with $\epsilon=0$ when $\omega_{p}=0$ and $\epsilon=1$ near optimal. See text for details.

In Fig. 3, we explore these issues within the context of our model. We consider three situations in which the Gaussian derived $T_{c}$ is plotted as a function of $\omega_{p}$ (which may be directly related to hole concentration $x$ ) to arrive at a form of "phase diagram". The solid line corresponds to the case in which the characteristic energy for pairing (called " $E_{F}$ ") is held fixed, and as observed in Fig. 11a, $T_{c}$ monotonically decreases. This should be contrasted with the situation in which $E_{F}$ is allowed to vary selfconsistently (dotted line) in accord with the measured $\omega_{p}$. Here for definiteness, we assume ${ }^{a}$ Luttinger volume Fermi surface. The latter case yield $\$ T_{c} \approx \omega_{p}$; however, $T_{c}$ is suppressed at low doping primarily as a result of the lowering of the electronic energy scale, $E_{F}$, rather than from increased phase fluctuations. Neither of the above cases is entirely satisfactory for understanding the larger pseudo-gap regime at low doping. We address this issue phenomenologically by introducing the effects of a dimensionality crossover for fixed pairing energy scale $E_{F}$ and calculating $T_{c}$ for a system in $2+\epsilon$ dimensions (dashed curve) where $\epsilon$ varies smoothly from zero when $\omega_{p} \approx 0$ (at half filling) to one when $\omega_{p} \approx 1.2 \mathrm{eV}$ (corresponding to optimal doping). Here $T_{c}$ is suppressed at low doping, as a consequence of $2 \mathrm{~d}$ fluctuation effects. Moreover, one can associate $T^{*}$ with the solid curve, which exhibits the observed experimental trends. Thus the low doping regime is characterized by a large pseudo-gap. On the other hand, at higher doping or $\omega_{p}$, both $T_{c}$ and $T^{*}$ converge and the pseudo-gap region vanishes. Whether or not this phenomenology is appropriate, the above discus- sion underlines the importance of multiple energy scales ( $\omega_{p}$, as distinct from the pairing energy scale) and the possible role of a dimensionality crossover 13 in understanding pseudo-gap behavior.

In summary, we have presented a pre-formed pair model in which the effects of Coulomb correlations are clearly seen to suppress superconducting fluctuations (above $T_{c}$ ) and thereby tune pseudo-gap behavior in the calculated specific heat and spin susceptibility. Moreover, these two thermodynamic variables, as a function of $T$, are found to be reasonably consistent with experiment. However, it should be stressed that within our microscopic model, the effects of Coulomb correlations enter in a rather different way than has been assumed in previous phenomenological schemes. 14 Our approach focusses more directly on correlated pairs rather than superconducting grains; therefore, phase and amplitude fluctuations appear on a relatively equivalent basis. As a consequence, introducing Coulomb interactions into the pre-formed pair formalism leads to a narrowing of the pseudo-gap region by providing a competing attraction in the particle-hole channel and thus reducing the effectiveness of superconducting pairing.

This work is supported by the National Science Foundation (DMR 91-20000) through the Science and Technology Center for Superconductivity. KL acknowledges the hospitality and support, via the National Science Foundation (PHY94-07194), of the Santa Barbara Institute for Theoretical Physics and JM greatfully acknowledges the financial support of the National Science and Engineering Research Council (Canada). Useful conversations with C. Castellani, M. Randeria, C. Sa de Melo and M. Tarlie are acknowledged.

${ }^{1}$ For a Review, see for example, Physics Today 49, 17 (1996).

2 See, for example, X. G. Wen, P. A.. Lee, Phys. Rev. Lett. 76, 503 (1996). H. Fukuyama, Prog. Theor. Phys. Suppl. 108, 287 (1992).

${ }^{3}$ P. Nozières and S. Schmitt-Rink, J. Low Temp. Phys. 59, 195 (1985).

${ }^{4}$ C, A, R, Sa de Melo et al, Phys. Rev. Lett. 71, 3202 (1993).

${ }^{5}$ V. J. Emery and S. A.. Kivelson, Nature 274, 434 (1995).

${ }^{6}$ R. Haussman, Phys. Rev. B 45, 12975 (1994).

${ }^{7}$ M. Randeria et al, Phys. Rev. Lett. 69. 2001 (1992).

${ }^{8}$ Y. J. Uemura et al, Phys. Rev. Lett. 62, 2317 (1989).

${ }^{9}$ L. Kadanoff and G. Baym, Benjamin (Reading) 1974.

${ }^{10}$ G. Mahan in "Many Particle Physics", Plenum (New York) 1981.

${ }^{11}$ D. Thouless, Annals of Physics 10, 553(1960).

12 J. O. Sofo and C. A. Balseiro, Phys. Rev. B 45, 8197 (1992).

13 J. W. Loram et al, Phil. Mag. 1405 (1992).

${ }^{14}$ S. Doniach and M. Inui, Phys. Rev. B 41, 6668 (1990). 